\title{
Routing Approaches for Wireless Sensor Network
}

\author{
Arun Kumar \\ Assistant Professor, \\ Department of Computer Engineering, \\ Maharishi Markandeshwar University, India
}

\author{
Vijay Kumar Katiyar, Ph.D. \\ Professor and Head, \\ Department Of Computer Engineering, \\ Maharishi Markandeshwar University, India.
}

\begin{abstract}
In this paper, we describe the approaches for routing in Wireless Sensor Networks. Their characteristics are discussed. An efficient routing approach is then proposed based on energy equations and use of advanced nodes having high energy than normal nodes. This intelligent cluster routing is then compared with SEP routing.
\end{abstract}

\section{General Terms}

Energy efficiency in Wireless Sensor Networks

\section{Keywords}

Flat Routing, Cluster Routing, Energy efficient approach for WSN

\section{INTRODUCTION}

A WSN consists of a large number of sensor nodes typically hundreds or thousands. Each sensor node comprises of different components which work together to act like sensor and can sense for some particular application. These units are:

a) Sensing unit:-it senses the events happening around it.

b) Processing unit:-it processes information to compute the results based on the sensed information by sensing unit.

c) Transmission unit:- it transmits the computed results to the base station.

d) Mobility monitoring unit: - monitors the movement of node to check whether it is mobile or stationary.

e) Position finding unit: - it finds the position of each node, which assists each node to calculate the distance from its neighbors.

f) Power Supply unit: - it provides energy to all components of the sensor node.

g) ADC: - converts the alternate current to direct current.

To improve the quality of service sensor nodes can communicate and coordinate with each other. Each sensor node has sensing, computational, and communicational capabilities. Each node has information which it has sensed or is provided by its neighbors. The quality of result which is produced by the sensor node depends upon the above said units. The processed result is then passed to all its neighbors or to the Base Station. Base Station is a node which queries for data to sensor node and collects the results from it. A base station can both be mobile as well as fixed.

\section{DESIGN CHALLENGES IN WSN}

One of the main reason which limits the use of sensors is its energy efficiency[1]. Because of small size of node, the battery size is also small which results in small lifetime of the sensor node. Sensor networks are designed for a specific applications. Some major issues in Wireless Sensor Networks are:-

1) Deployment of nodes in the network: - here the position of the node in the network is dealt with. The position may be predetermined or it may be decided on the spot.

2) Use of energy resources and data accuracy: - A node depends much upon its energy resource. If the energy is low, the processing speed of a sensor will also be low and the result produced by it gets affected. After calculating the result node transmits the data to the base station. But if the power goes below the threshold there will be no transmission.

3) Type of data reporting: - Data reporting can be a) Time driven where computed result is passed to base station after a fixed interval of time. b) Event driven where the result is reported only after the occurrence of a particular event. c) Query driven in which result is passed to sink only after getting a particular query from the sink. d) Hybrid which is a combination of all above defined schemes.

4)Heterogeneity in the sensor network: - Some routing protocols in WSN were based on assumption that all nodes have equal capability and efficiency. But the assumption is not true for every case. Nodes may differ in their efficiencies.

The presence of this heterogeneity leads to many technical difficulties.

1) Fault Tolerance:-Sensor nodes are prone to faults. The node may fail because of lack of power supply, so the network design must be of distributed type.

2)_Coverage area:- Most type of sensors cover only small area due to tight coupling. This is one major hurdle in design of a sensor network.

\section{ROUTING PROTOCOLS IN WSN}

Three main classes of routing protocols are:-

1) Proactive: - Routes from every node to the base station are predefined.

2) Reactive:- Routes from each node to the base station are computed when there is a demand for the route.

3) Hybrid: - Some routes are computed in advance and some are defined after the demand is raised.

\section{OTHER GENERAL CLASSIFICATIONS}

Based on the structure of the network and Based on the operation of the protocols routing protocols are categorized. 


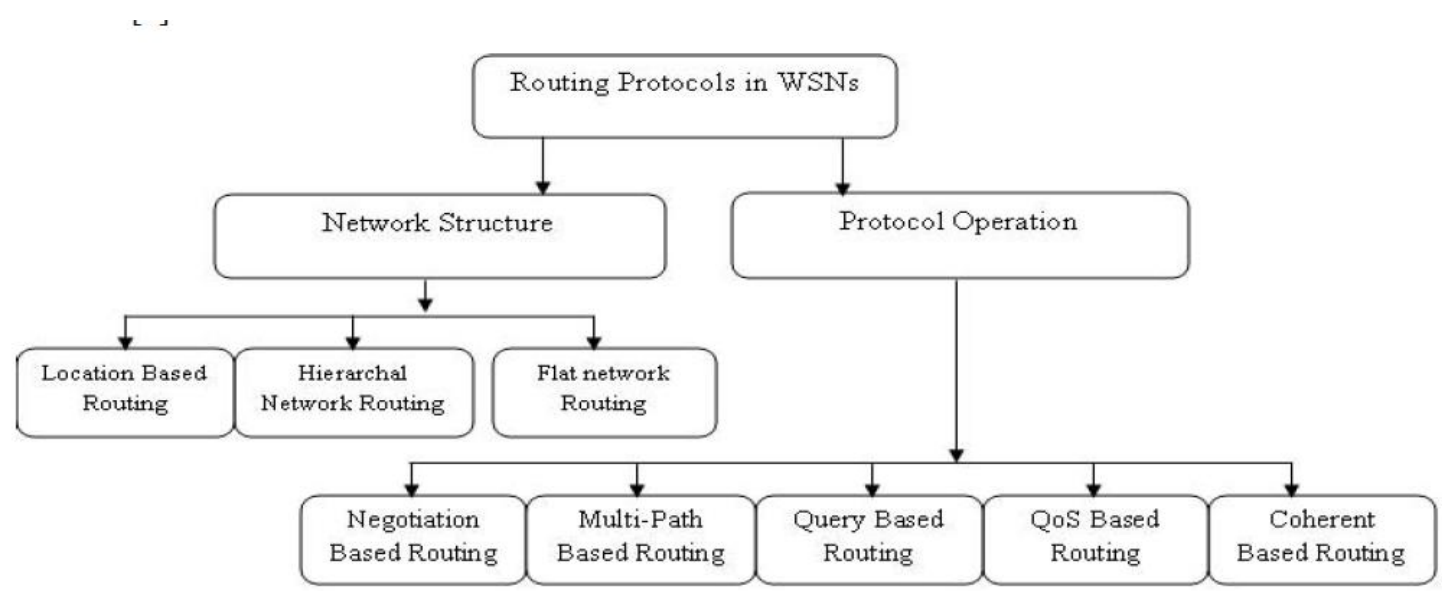

Figure 1 Classification of routing protocols

In this paper, we have mentioned Flat routing, Multi-hop routing and Hierarchical routing

\subsection{Flat Routing}

In flat routing each node of the network is assigned the same task. To produce the final result different nodes with same tasks co-operate with each other. Because of the large number of nodes global addressing is not possible and the result is passed on the basis of some query. This technique of dispatching result according to query is known as date-centric approach as the data is dispatched according to some attributes. For example [Temp $60 * \mathrm{~F}$ ], only the node which senses the temperature $>60 * \mathrm{~F}$ transmits the result to BS. There are many protocols which fall in this category.

\subsubsection{Sensor Protocol for Information via Negotiation (SPIN)}

In SPIN family of protocols the information is passed to all nodes in the network on the assumption that each node has equal capability of computation \& communication [5]. This class use data-negotiation \& resource adaptive algorithms. SPIN collects Meta- data from different nodes; a unique ID may be given to this Meta- data for avoiding redundant data. SPIN is a Periodic protocol means it works on the basis of time, produce result after a particular period of time, whether or not there is a need of data.

\section{Two Basic Ideas of Design:-}

1. To save the energy it only sends the sensed data instead of sending all the information.

2. It overcomes the disadvantage of flooding \& gossiping protocols which is redundancy of data because of overlapping ranges of neighboring nodes.

SPIN protocol is a three-phase protocol which works in three steps. These steps are in the form of messages. i)ADV(Advertisement), ii) REQ- (Request), iii) DATA- (Original Content).The working starts with the advertisement phase.

ADV: - The sensor node in the network which have some new data to share, advertize that data to its neighboring nodes with the help of ADV messages.
REQ: - After listening to the ADV message the neighboring nodes came to know about the data of their interest from the advertisement. If no data of their interest is found, they avoid the ADV message otherwise data is requested by the node using the REQ message.

DATA: - After getting the request message from the neighbor the sender node sends the data packet to that node. This packet is identified by a unique id.

SPIN2 is another protocol of spin family which is a resource awareness protocol. It uses a lower limit of energy known as threshold value, if the energy level is below this threshold value the protocol denies to participate in the communication, but if the energy level is greater than the threshold it works using the above mentioned three phases. SPIN provides better efficiency than the flooding and gossiping protocols.

\subsubsection{Direct Diffusion}

In WSN Direct diffusion is known for its data aggregation operation. It is Data-Centric communication based application oriented protocol. Here attribute-value pair is used to represent the result from sensor and the query from the base station. In this protocol energy of the sensor nodes is preserved with the help of aggregation mechanism. It collects the result from the different sensor nodes, aggregates the result, remove the redundant data to minimize the number of transmissions of same data repeatedly, which as a result reduce the energy consumption of the network. Direct Diffusion is a multiple-source-single-sink protocol, which defines the path from multiple sensor nodes to single base station. The BS sends the query to sensor nodes by broadcasting which contains the data of interest.

Interest: - It is the task which the BS requires and is accomplished by the sensor nodes. The interest is different for each sensor node, and each node forwards its interest to its neighbors. The interest moves from node to node throughout the network and the nodes through which the interest passes forms the gradients of the interest. 


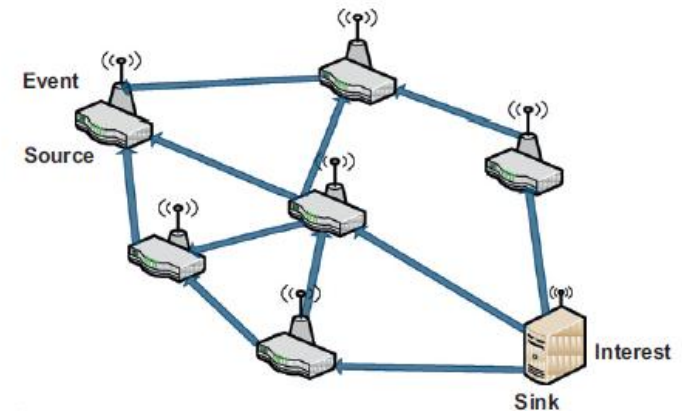

b

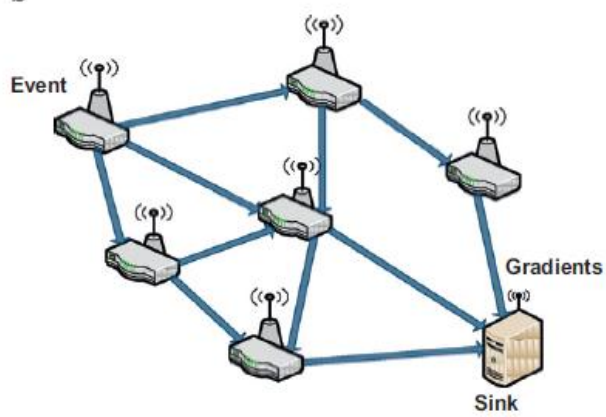

Figure 2. Formation of interest and gradients

Gradient: - It is an attribute-value pair and is based on the interest requested by the base station. A direction vector is associated with this attribute-value pair to give the direction of movement of the gradient. These gradients are formed from source to destination. Multiple paths are defined for all sensor node which participate in the interest of the sink (BS).

Data dissemination: - After paths are set from the source to the destination with the help of gradients, the least cost path is selected and data is transferred from source to the sink

BS refreshes itself after a particular period of time and sends the interest again to the network. This is repeated until it gets the full information.

\subsubsection{Rumor Routing}

Rumor routing is an alternative approach to direct diffusion[1]; it also contributes to the energy conservation of sensor nodes. The algorithm is based on the agents and events. The algorithm queries for the data only to nodes which know about the data instead of flooding the whole network. Broadcast is based on the events. An event is a small amount of change in the surrounding of a sensor node. Each node has an event table where record of all the events which have been sensed by the node is kept. When a node observe an event around it, it checks the event table, if the same event has already been listed no change takes place. Iif there is some new event then the node updates the events table.

Based on the event the node generates a data packet known as "Agent". This agent is then forwarded to all neighbors of the node which has generated the agent. The neighbor nodes adds the agent to their events table along with the identification of the node from which the event has came. BS queries to those nodes only that have generated the agents in the network instead of flooding the whole network. Nodes which has result related to the query immediately respond to the BS. Unlike direct diffusion where multiple paths have formed from source to destination there is only one path from source to destination. The main drawback of the protocol is the consumption of energy in maintaining the events table.

\subsubsection{Minimum Cost Forwarding Algorithm (MCFA)}

This algorithm is very simple which is similar to the Distance Vector Routing algorithm of Ad-hoc networks [29]. This algorithm is based on the assumption that two ends, source and destination of the network are always fixed at their locations, and we only have to find a path from source to the destination. Instead of events table, the routing table is maintained which contains the least cost path from source to destination. Each node proactively determines the least cost path from source to destination and store this path to its routing table.

BS broadcasts the message to all sensor nodes of the network. In the network each node and the link through which they are connected assumed to have some cost associated with them which helps to calculate least cost. The message broadcasted by the BS have initial cost set to zero and every other node in the network have initial cost set to infinity. When a query message reaches to a node, it calculates of costs associated with the message and link from which it has been received. If the calculated cost is less than the estimated cost, the cost at the node and the cost associated with the message are updated.

There is a drawback of this algorithm, a number of updates may take place within a second all having the same cost, then it is difficult to choose the best path. To prevent this problem a back-off algorithm was designed which makes sure that the update will take place only after a certain amount of time that is $\mathrm{C} * \mathrm{Lc}$, where $\mathrm{C}$ is some constant and $\mathrm{Lc}$ is the cost associated with the link from where last update have been received. After getting the query message a node checks whether or not it has the least cost path to the destination. If it has it sends the result to the destination, but if not then it rebroadcasts the message to its neighbors, this process is repeated till a path to destination has been discovered.

\subsubsection{Gradient Based Routing (GBR)}

GBR is also an enhancement of direct diffusion [1]; its working is same as of MCFA which maintains the least cost path. Likewise GBR has to track the number of hops through which the interest has propagated in the network.

Some terms of interest in this scheme:

Height of node: - It is the minimum number of hops which a packet passes from a node to base station.

Gradient:- The difference between the height of a node to that of its neighbor is considered as gradient. A packet which travels from source to destination is forwarded on the path whose gradient is largest.

Relay node: - Relay node is the crossing point from which multiple paths to the destination have been passed.

Three basic schemes of gradient based routing:-

a) Stochastic scheme: - There may be a possibility that two or more links have the same gradient. The problem is to choose one of these with similar gradients. According to stochastic scheme the node selects any instant gradient to forward the packet.

b) Energy based scheme: - In this scheme when the energy level of the node goes below the threshold value the node increases its height to increase its gradient. 
c) Stream based scheme:- The nodes which are busy in routing some stream of data are not considered for the route of some other scheme.

The above defined schemes are used in a multiple path algorithm.

\subsubsection{Information Driven Sensor Querying (IDSQ) and Constrained Anisotropic Diffusion \\ Routing $(C A D R)$}

The principle of this algorithm is to route the data in the network in such a way so that information gain is maximized and total delay is minimized. Some criterion is applied to information before querying the information. BS first decides an event for which it is going to query, then it checks out which node are near to that specific event area. Only the nearby nodes in the area of interest are used for routing and the routes from active nodes to the base station are updated periodically.

CADR allows each node to estimate a cost per information gradient, and the information which cost less and sufficient for use is then forwarded to the sink node. In addition to CADR, the IDSQ also finds the nodes which can be more beneficial to the user i.e. which can provide additional and significant information based on the query. It selects a single node from those which are selected by CADR and establishes a path from that node to the sink.

\subsubsection{Cougar}

The basic idea of COUGAR is to hide the query processing from the network layer operations. To achieve this objective of abstraction COUGAR use declarative queries i.e. queries which can explain itself to the network. These queries have been already declared in an additional layer called query layer which is put in between the network layer and the application layer.

COUGAR uses inbuilt data aggregation function of the network. From different nodes in the network a node with the higher computational power is selected as the leader node, whose task is to perform data aggregation and data transmission. BS provides a leader selection and route management mechanisms to network nodes. BS refreshes the query periodically and with this leader node and route information are also updated. Main drawback of COUGAR is implementation of an additional layer, the query layer which consumes extra energy.

\subsubsection{Active Query Forwarding In Sensor Network}

Active Query forwarding in Sensor Networks [30] AQUIRE algorithm uses the concept of distributed database and sub queries. The more complicated and large queries are decomposed into small, simple and easily manageable sub queries.

Each node in the network has some cached information. BS sends sub-queries to the sensor nodes upon receiving the subquery each node first checks its cache for a solution. If it has some information about the query it attaches the information with the query and forwards the packet to its nearest neighbor which is within the boundary of the network. If a node does not have any information it then collects that from its neighbors. There is an aggregator node whose task is to collect the information from different nodes and forwarding the result to the base station.

\subsubsection{Energy Aware Routing}

The main objective of the protocol is to minimize the energy consumption of a sensor node in its basic operations for increasing the life time of the sensor node. It looks like direct diffusion but is different in that it is responsible for providing a set of optimal paths from source to destination rather than providing single optimal path. The determination of the set of optimal paths is done by a probabilistic mechanism. The low a path consumes the energy increased is its probability to be added in the optimal path set.

Now work is being carried out on power-aware routing protocols for wireless networks [39, 40, 41]. Here in these protocols, optimal routes are selected based on the energy at each node along the route. Routes that are longer but use nodes with more energy than the nodes along the shorter routes are given preference. This approach avoids hot-spots in the network, where a node is used frequently to route other node's data, and it helps in distributing energy dissipation evenly.

One method of choosing routes is to use minimum transmission energy (MTE) routing [35, 36]. In this approach, the intermediate nodes are chosen in such a way so that the sum of squared distances (and hence the total transmission energy, assuming a $\mathrm{d}^{2}$ power loss) is minimized; therefore for the configuration shown in Figure 1, node A would transmit to node $\mathrm{C}$ through node $\mathrm{B}$ if and only if:

$\mathrm{E}_{\text {transmit }}\left(\mathrm{d}=\mathrm{d}_{\mathrm{AB}}\right)+\mathrm{E}_{\text {transmit }}\left(\mathrm{d}=\mathrm{d}_{\mathrm{BC}}\right)<\mathrm{E}_{\text {transmit }}\left(\mathrm{d}=\mathrm{d}_{\mathrm{AC}}\right)$ (1)

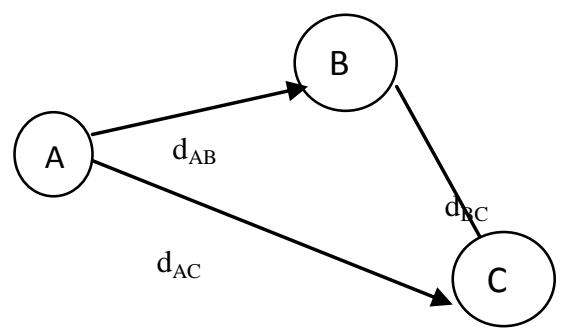

Figure 1 : Nodes for MTE

MTE runs a start-up routine to determine its next-hop neighbor. This approach of choosing routes minimizes the transmit energy required to feed the data to the base station. Data are passed to each node's next-hop neighbor until the data reaches the base station. When nodes run out of energy the routes are determined again to ensure connectivity with the base station.

The working of EAR protocol is based on the assumption that each node in the network can be given an address to represent its type and position in the network. The query is flooded into the network to establish a communicational connection. Once the connection is established all the possible routes from source to destination are determined.

Now each route is chosen and its energy cost is calculated, and the path with lowest energy cost is added first to the optimal path set. By repeating the same process the optimal path set is completed. Each node maintains a forwarding table which assists it to forward data to its neighbors through the optimal path. 


\subsection{Multi-Hop Routing}

Routing protocols for networks connected with wire falls into two classes: distance vector routing and link-state [42]. Distance vector gets route packets by having each node pass distances to its neighbors, who then selects the shortest path to a given destination and store this information in a routing table. When packet come to the node, the node sees its routing table to get the next hop node to send the packet to its destination. In link-state protocol nodes spread the entire topology map and individual nodes use a shortest path algorithm (like Dijkstra's Algorithm) to find the best path to a given destination. These routing methods are used in wireless networks with little modifications, resulting in destinationsequenced distance vector (DSDV) and ad hoc on-demand distance vector (AODV) routing protocols.

But there are problems with using these routing approaches in wireless networks. The periodic messages needed to maintain valid routes may not only increase the traffic on the network, they may also deplete the limited battery power of a portable node. Dynamic source routing (DSR), handles this by creating routes on an on-demand basis [14]. This reduces the amount of efforts required for creating routes, at the expense of latency in searching a route when it is required. These are adhoc, self-configuring protocols, which handles node failures effectively.

Work has been done on "minimum-energy" routing protocols to extend the lifetime of the small devices in a wireless network. Like, there is an approach for selecting multi-hop paths to reduce the power dissipated in the nodes along the path. In these techniques, an intermediate node is used as a hop if and only if it minimizes the total energy compared with without using this middle hop node. In another similar approach, the authors observe that in a wireless network, data communication between neighboring nodes causes interference, which can reduce performance. Hence, they selected paths to minimize energy dissipation subject to a minimum interference criterion.

\subsection{Hierarchical Routing}

Hierarchical routing is also known as cluster based routing[3]. In this type of routing the nodes with higher energy are used for computational and communicational purpose, while the nodes with low energy are used for sensing purpose. It is more efficient technique as compared to flat routing. Data is aggregated at cluster level which decreases the number of packets being sent from source to destination, which in turn decrease the consumption of energy. Use of cluster heads is an important feature of hierarchical routing.

In terms of cluster formation there are two different approaches. In one approach, each node broadcast in a certain region its properties (id, node degree, residual energy etc.), after which an election process is executed to choose the cluster head [37], [38]. This approach generally assures regular cluster size and full node coverage, but at the cost of high communication overhead. In another approach, the clustering algorithm is triggered at regular intervals to select new cluster heads. At each interval, the clustering process uses a certain number of iterations to finally get the desired cluster head. If the minimum probability of a node becoming a cluster head is $p$, it takes $N<=\left[\log _{2} 1 / p\right]+1$ steps for the election algorithm to stop $(N=6-15$ iterations for average scenarios). During each iteration, a designated cluster head generates broadcasting messages, resulting in a total message cost (for setting up the cluster ) to be of order $N * n$, where $n$ is the total number of nodes in the network. This results in significant energy consumption, given that the election process is called repeatedly to achieve load balancing.

Another approach, such as the one used in [11], is to specify a certain probability for each node to become a cluster head, and the node which turns out to be cluster head announces itself through flooding. This approach has lower message overhead, but cannot assure uniform cluster head distribution and complete coverage of all non-cluster nodes. Therefore, the nodes not covered by any cluster heads have to send their messages directly to the sink, significantly increasing the total message cost.

Since cluster heads require more energy for communication and computation, cluster head rotation is an important step of any clustering scheme. For most of the clustering schemes, rotation involves re-election of cluster heads, which requires a lot of efforts. With well designed rotation sequence, the role of cluster heads can be uniformly distributed to all sensors in the network.

\section{CONCLUSION}

In flat routing each node of the network is assigned the same task. Different nodes with same tasks co-operate with each other to give the final result.

Whereas in hierarchical routing the nodes with more energy are used for computation and for communication, while the nodes with low energy are used for sensing purpose. It is more efficient technique as compared to flat routing.

\section{ACKNOWLEDGMENTS}

Our thanks to the staff of Computer Engineering Department, Maharishi Markandeshwar University who provided needed resources for the mentioned work.

\section{REFERENCES}

[1] M. Palit and R.C. Biradar "A Survey on Routing Protocols in WSN", Networks (ICON), $18^{\text {th }}$ IEEE International Conference, Dec. 2012,

[2] Rathna and Sivasubramanian, "Improving Energy Efficiency in Wireless Sensor Networks through Scheduling and Routing", Research Scholar, Sathyabama University, TamilNadu, India, International Journal of Advanced Smart Sensor Network Systems, vol: 2, pp: 21-27, January 2012.

[3] Shio Kumar Singh, M P Singh, D K Singh, "A Survey of Energy-Efficient Hierarchical Cluster-Based Routing in Wireless Sensor Networks", International Journal. of Advanced Networking and Applications, vol: 02, pp: 570-580, 2010.

[4] Honey Soni, Priyanka Tripathi and Robin Singh Bhadoria, "An Investigation on Energy Efficient Routing Protocol for Wireless Sensor Network", Computational Intelligence and Communicational network, vol: 7, pp: 141 - 145, Sept. 2013.

[5] Deepa H, Aayan Kumar Daas ," A Study on Routing Protocols in Wireless Sensor Network", published in: International journal of computer applications, vol: 72,pp-35-39, 2013.

[6] Seyedeh Zahra Yazdanpanah, Yousef Abbasnejad Varzi, Ali Haronabadi, "An Improved energy Consumption Method For WSN", Fuzzy Statement and Knowledge Discovery, vol:-4, pp:-1123-1132, 2014. 
[7] Li Ya, Wang Pengjun, Luo Rong, Yang Huazhong, Liu Wei," Reliable Energy-Aware Routing Protocol for Heterogeneous WSN Based on Beaconing", Advance Communication Technology(ICACT), vol:-8, pp:109112 , Feb 2014.

[8] Al-Karaki, J.N, "Routing Techniques in WSN: A Survey", Wireless Communications, IEEE, vol: 11, pp: 6-28, Dec. 2004.

[9] Jia Xu,Ning Jin, Xizhong Lou,Ting Peng,Qian Zhou, Yanmin Chen, "Improvement of Leach Protocol for WSN", Fuzzy System and Knowledge Discovery(FSKD), vol:-11, pp:-2174-2177 May. 2012.

[10] Amis, A. D., Prakash, R., Huynh, D., and Vuong, T., Max-Min D-cluster Formation in Wireless Ad Hoc Networks," in Proceedings of The 19th Conference on Computer Communications(INFOCOM'00), (Tel-Aviv, Israel), pp. 32-41, 2000.

[11] Bandyopadhyay, S. and Coyle, E. J., An Energy Efficient Hierarchical Clustering Algorithm for Wireless Sensor Networks," in Proceedings of The 22nd Conference on Computer Communications(INFOCOM'03), (Sanfrancisco, CA), pp. 1713 - 1723, 2003.

[12] Chunyao Fu, Zhifang JIANG, Wei WET, "An Energy balanced Algorithm of Leach Protocol in WSN", International Journal of Computer Science, vol:-10, pp:354-359, 2013.

[13] Yun Li, Nan Yu, Weiyi Zhang, Weiliang Zhao, "Enhancing the Performance of Leach Protocol for WSN", IEEE INFOCOM, pp:-223-228, 2011.

[14] Laveena Mahajan "Improving the Stable Period of WSN using Dynamic Stable Leach Election Protocol" Issues and Challenges in Intelligent Computing Techniques (ICICT), vol-11, pp: 393-400, 2014.

[15] Huu Nghia Le, Vyacheslav Zalyubovskiy, Hyunseung Choo "Delay-minimized Energy-efficient Data Aggregation in Wireless Sensor Networks", International Conference on Cyber Enabled Distributed Computing and Knowledge Discover (IEEE), vol-1, pp: 401-407, Oct. 2012.

[16] Linlin Wang, JieLiu, Wei Wang "An Improvement and Simulation of LEACH Protocol for Wireless Sensor Network", First International Conference on Pervasive Computing, Signal Processing and Applications (IEEE), pp: 444-447, Sept. 2010.

[17] Ma Chaw Mon Thein, ThandarThein "An Energy Efficient Cluster-Head Selection for Wireless Sensor Networks", International Conference on Intelligent Systems, Modeling and Simulation (IEEE), vol:-8, pp.287-291, Jan. 2010.

[18] Meenakshi Sharma and Anil Kumar Shaw "Transmission Time and Throughput analysis of EEE LEACH, LEACH and Direct Transmission Protocol: A Simulation Based Approach", Advanced Computing: An International Journal (ACIJ), vol: 3, pp: 5-9, September 2012.

[19] M M Islaml, M A Matin2, T K Mondol 1 "Extended Stable Election Protocol (SEP) for Threelevel Hierarchical Clustered Heterogeneous WSN", (IEEE), vol: 5, pp. 1-4, June 2012.
[20] Reetika Munjal, Bhavneesh Malik "Approach for Improvement in LEACH Protocol for Wireless Sensor Network", Second International Conference on Advanced Computing \& Communication Technologies (IEEE), pp.517-521, Jan. 2012.

[21] B.A. Sabarish, R. Lavanya,'Modified Leach Protocol for WSN", International Journal of Computer Applications, vol:-62, pp:-1-4, 2013.

[22] Shuo Shi, Xinning Liu and XuemaiGu "An EnergyEfficiency Optimized LEACH-C for Wireless Sensor Networks", $7^{\text {th }}$ International ICST Conference on Communications and Networking in China (CHINACOM) (IEEE), vol:5, pp: 487-492, Aug. 2012.

[23] Smaragdakis. Georgios. Ibrahim Matta. And Azer Bestavros. "SEP: A stable election protocol for clustered heterogeneous wireless sensor networks", Boston University Computer Science Department, pp: 223-230, 2004.

[24] Thu Ngo Quynh 1, Kieu-Ha Phung2,3, Hoan Vu Quocl, "Improvement of Energy Consumption and Load Balance for LEACH in Wireless Sensors Networks", published by:(IEEE), vol:73, pp.583-588, Oct. 2012.

[25] Vivek Katiyar, Narottam Chand, Gopal Chand Gautam, Anil Kumar "Improvement in LEACH Protocol for Large-scale Wireless Sensor Networks", (IEEE), vol:32, pp:-1070-1075, Mar. 2011.

[26] Yuhua Liu Yongfeng Zhao Jingju Gao "A New Clustering Mechanism Based On LEACH Protocol", International Joint Conference on Artificial Intelligence (IEEE), Hainan, pp.715-718, April 2009.

[27] Shijun He, Yanyan Dai, Ruyan, "A Clustering Protocol for Energy Balance of WSN based on Genetic Clustering Algorithm", International Conference on Future Supported Education, pp:-788-793, 2012.

[28] Fuzhe Zhao, You Xu, Ru Li,"Improved Leach Communication Protocol for WSN", International Conference on Control Engineering and Communication Technology, pp:-700-702, 2012.

[29] Bhaskar Krishnamchari, Deborah Estrin,'Impact of Data Aggregation in WSN", IEEE Journal Selected Areas in Communications, pp. 1333-36, Aug.2009.

[30] M. Chu, H. Haussecker, and F. Zhao, "Scalable Information-Driven Sensor Querying and Routing for ad hoc Heterogeneous Sensor Networks," published in The International Journal of High Performance Computing Applications, Aug. 2002, Vol. 16, pp: 106-109.

[31] S. Lindsey, C. Raghavendra, "PEGASIS: PowerEfficient Gathering in Sensor Information Systems", published in: IEEE Aerospace Conference Proceedings, 2002, Vol. 3, pp. 1125-1130.

[32] Yamunadevi, S.P,"Efficient Comparison of Multipath Routing Protocols in WSN", Computing Electronics and Electrical Technologies (ICCEET), vol: 2, pp: - 807-811, mar. 2012.

[33] Jianguo SHAN, Lei DONG, "Research on Improved Leach Protocol of WSN”, IEEE, pp:-75-77, 2013.

[34] Younis, O. and Fahmy, S., HEED: A Hybrid, Energy efficient, Distributed Clustering Approach for Ad-hoc 
Sensor Networks," IEEE Transactions on Communications, vol. 3, no. 4, pp. 366-379, 2004.

[35] M. Ettus, "System Capacity, Latency and Power Consumption in Multihop-Routed SS CDMA Wireless Networks", Radio and Wireless Conference (RAWCON'98), pp.55-58, August 1998.

[36] T. Shepard, "A Channel Access Scheme for Large Dense Packet Radio Networks", ACM SIGCOMM, pp.219-230, August 1996.

[37] "Multiplexing Protocol for Low Bitrate Multimedia Communication" ,ITU-T Recommendation H.223, September 1997.

[38] M. Bhardwaj and A. Chandrakasan, Power Aware Systems, $34^{\text {th }}$ Asilomar Conference on Signals, Systems and Computers, November 2000

[39] X. Lin, I. Stojmenovic, "Power-Aware Routing in AdHoc Wireless Networks", SITE, University of Ottawa, TR-98-11, December 1998.
[40] S. Park, M. Srivastava, "Power Aware Routing in Sensor Networks using Dynamic Source Routing" , ACM MONET, Special Issue on Energy Conserving Protocols in Wireless Networks, 1999.

[41] S. Singh, M. Woo, C.S. Raghavendra, "Power Aware Routing in Mobile Ad Hoc Networks", Proceedings of the Fourth Annual ACM/IEEE International Conference on Mobile Computing and Networking (Mobicom '98), October 1998.

[42] T. Shepard, "A Channel Access Scheme for Large Dense Packet Radio Networks, ACM SIGCOMM, pp.219-230, August 1996.

[43] http://citeseerx.ist.psu.edu/viewdoc/summary?doi=10. 1.1.129.2369

[44] http://ijrts.com/Downloads1.php?file=140112002 